Voix et Images

voixetimages

\title{
Expression et expansion : l'offensive littéraire française de l’après-guerre au Québec
}

\section{Roland Houde et Jacques Beaudry}

Volume 14, numéro 2 (41), hiver 1989

L’édition littéraire au Québec

URI : https://id.erudit.org/iderudit/200772ar

DOI : https://doi.org/10.7202/200772ar

Aller au sommaire du numéro

Éditeur(s)

Université du Québec à Montréal

ISSN

0318-9201 (imprimé)

1705-933X (numérique)

Découvrir la revue

\section{Citer cet article}

Houde, R. \& Beaudry, J. (1989). Expression et expansion : l'offensive littéraire française de l'après-guerre au Québec. Voix et Images, 14(2), 237-247.

https://doi.org/10.7202/200772ar d'utilisation que vous pouvez consulter en ligne.

https://apropos.erudit.org/fr/usagers/politique-dutilisation/ 


\title{
Expression et expansion: l'offensive littéraire française de l'après-guerre au Québec
}

\author{
par Roland Houde, Université du Québec à Trois-Rivières \\ et Jacques Beaudry, Université de Sherbrooke
}

La discontinuité est en effet la loi de Babel où la seule faille du sexe est parfaitement continue de bas en haut sous les replâtrages divers de l'amour et de l'art, eux-mêmes diversement compris selon les époques et les étages des siècles.

Pierre Trottier, Mon Babel, Montréal, HMH, 1963, p. 14.

Il existe plusieurs représentations du rôle de suppléance, d'émulation, voire de concurrence, que certains jeunes intellectuels du Canada français décidèrent de jouer pendant la Seconde Guerre mondiale afin de maintenir et de promouvoir la littérature francophone ici et ailleurs ${ }^{1}$. Mais il y a aussi une réfraction étrange que Pierre Tisseyre, alors président du Conseil supérieur du livre du Québec, a livrée dans les Nouvelles littéraires de Paris en 19712.

Sans doute pour les besoins de sa cause et de ses intérêts, peu soucieux de sa situation dans la continuité historique du développement progressif de l'édition canadienne-française depuis le $\mathrm{XIX}^{\mathrm{e}}$ siècle, Pierre Tisseyre répond ainsi à la question posée par le chroniqueur-enquêteur Jean Montalbetti, «Comment est née l'édition québécoise après-guerre?»:

L'édition québécoise est née pendant la guerre comme une relève nécessaire de l'édition française dont les livres ne parvenaient plus de ce côté de l'Atlantique. Mais ces éditeurs, qui s'étaient mis à publier librement des auteurs français: Bernanos, Mauriac ou Georges

1 Pour mémoire, rappelons quelques-unes de ces versions: Rex Desmarchais, France immortelle, Montréal, Éditions Libres, 1941; Robert Charbonneau, la France et nous, journal d'une querelle, Montréal, l'Arbre, 1947; René Garneau, les Littératures contemporaines à travers le monde, Paris, Hachette, 1961, p. 287288; Germaine Brée et Édouard Morot-Sir, Littérature française, 1984, t. 9, p. 39; Jacques Michon, «Édition littéraire et autonomie culturelle, le cas du Québec», Présence francophone, no 26, 1985, p. 58-60, ainsi que «Croissance et crise de l'édition littéraire au Québec (1940-1959)», Littérature, no 66, 1987, p. 115-126.

2 No 2303, 12-18 novembre 1971, p. 11 «Un éditeur vous parle Pierre Tisseyre: Le Québec en quête d'auteurs». 
Duhamel, ont tous fait faillite en 1945 quand le livre français reprit ses droits. Toutes ces faillites avaient alarmé les banques qui refusaient désormais de consentir des prêts à l'édition. Je crois pouvoir dire que dans ces circonstances j' ai fait cuvre de pionnier en lançant la littérature canadienne-française. En effet, pendant très longtemps, j'ai publié les $2 / 3$ des romans québécois. Depuis, d'autres éditeurs ont ouvert leurs portes après moi.

Réponse pour le moins édifiante dont les entours relatent brièvement les origines françaises de l'interviewé, ses études, son œuvre ${ }^{3}$, publiée sous l'occupation, son implication à New York dans la création d'une branche canadienne du Cercle du livre de France (avec sans doute des fonds franco-américains) 4 . Nous savons par ailleurs que, en 1946, M. Tisseyre est rédacteur en chef de l'édition américaine de la revue le Monde français de Paris et Montréal ${ }^{5}$, en faveur des «beaux livres français» et des «collections soignées» des Éditions Variétés ainsi que de «la principale revue d'expression française en Amérique», la Nouvelle Relève, fondée en 1934, dirigée par Robert Charbonneau et Claude Hurtubise. Soit. Examinons de plus près cette partie de la réponse de $\mathrm{M}$. Tisseyre: «Mais ces éditeurs, qui s'étaient mis à publier librement des auteurs français: Bemanos, Mauriac ou Georges Duhamel, ont tous fait faillite en 1945 quand le livre français reprit ses droits».

Parmi «ces éditeurs», il faut bien compter les Éditions de l'Arbre de Charbonneau et Hurtubise qui publièrent Bernanos... librement, avec ferveur et contre rémunération. Et ce, nous le savons, depuis le premier texte de Bernanos, «Redevenir humain» publié dans la Nouvelle Relève en septembre 1941. Le «cas» Bernanos est mal choisi, de toute évidence. De fait, la Lettre aux Anglais de Bernanos fut une aventure conjointe entre l'éditeur brésilien, Charles Hofer dit Ofaire (Atlantica Editora) et l'éditeur canadien, Hurtubise-Charbonneau (l'Arbre), comme en témoigne la justification de première édition de février 1942: « 6,000 exemplaires dont 2,000 pour les Éditions de l'Arbre à Montréal» en plus de la page couverture. La deuxième édition de cette Lettre... est datée de janvier 1943 et la troisième (15e mille) de juillet 1943, mais étrangement, ces éditions de 1943 ne comportent plus la mention de l'Arbre. Selon nous, cette étrangeté s'explique par le contrôle de la diffusion, du point de distribution de la littérature francophone (grande ou petite) en Amérique et ailleurs: Montréal versus New York. Elle n'a rien à voir avec les valeurs de suppléance, de complémentarité culturelle, de fraternité française. Ce projet se met en place dès 1942-43 à New York sous la direction de la Librairie de France et de sa filiale,

355 Heures de guerre, Flammarion, 1943, avec réimpression canadienne par le Cercle du livre de France de New York et Montréal en 1947.

4 Tous ces renseignements se retrouvent complétés dans le Dictionnaire pratique des auteurs québécois de Réginald Hamel, John Hare et Paul Wyczynski (Fides, 1976), p. 653.

5 Mensuel fondé par Albert et Paul Duchêne et imprimé à Saint-Jérôme, sous la direction générale de C.-A. Le Neveu, avec page publicitaire ( $2^{\mathrm{e}}$ année, vol. III, no 10 , juillet 1946). 
les Éditions de la Maison Française localisées à la même adresse: 610 Fifth Avenue ${ }^{6}$.

Malgré ces faillites, certaines maisons se sont implantées ici à la fin des années 40 alors que d'autres continuaient d'opérer malgré la prolifération d'établissements du même genre à New York avec ou sans le support d'une Direction générale des relations culturelles, d'une puissante Société des Gens de lettres, d'une très efficace Commission nationale du livre français à l'étranger présidée par Édouard Herriot (aussi président de l'Assemblée nationale de France), assisté par Georges Duhamel et Paul Montel à la vice-présidence, dont le mandat, d'àprès le décret constitutif, était:

a) d'étudier toute mesure ayant pour but d'améliorer la diffusion du livre français;

b) de soumettre ses conclusions au gouvernement avant le $1^{\text {er }}$ novembre 1948;

c) de donner son avis sur les questions concernant la diffusion du livre français à l'étranger qui lui seront soumises par les ministres intéressés.

Parlementaires français, savants et écrivains, professionnels de l'édition étaient représentés à cette commission dont Georges Bidault, ministre des Affaires étrangères; Julien Cain pour le ministère de l'Éducation nationale; Maurice Bedel, Jean Cassou, Jules Romains, Mario Roques, Vercors pour les personnalités; Étienne Gilson et Paul Rivet pour les parlementaires; MM. Angoulvent, Hartmann, Rousseau et Seghers pour les professionnels 7 . Selon nous, cette convergence ou concertation d'efforts représentait une des réponses à l'appel du ministre de l'Éducation nationale, Marcel Naegelen, lors de sa «Conférence» devant les ambassadeurs en poste à Paris le 2 avril 1946 au

6 À preuves: p. 25, no 41, 1943, de la revue Voici la France de ce mois; vol. I, no 1, mai 1943 de cette autre revue, le Monde libre, publiée par l'Arbre et imprimée par l'Imprimerie du Sacré-Cœur de Laprairie, mais dont les livraisons seront, par la suite, imprimées à New York (cf. vol. II, no 4, novembre 1944 ou vol. II, no 5 , décembre 1944-janvier 1945). Dans cette demière livraison (p. 97), on peut voir que le projet s'est concrétisé effectivement: le Monsieur Ouine, tant désiré par l'Arbre, est distribué par les Éditions de la Maison Française de New York. Faibles preuves, dira-t-on? Il en existe cependant une autre plus forte. Cette preuve se retrouve depuis 1971 consignée dans le tome II (1934-1948) de la Correspondance de G. Bernanos, recueillie par Albert Béguin, choisie et présentée par sour Jean Murray, o.p., et publiée chez Plon. Elle concerne M. Crespin de la Librairie de France et des Editions de la Maison Française de New York qui dès la fin de 1941 entre en contact avec l'éditeur brésilien de Bernanos et se présente comme «éditeur canadien» (cf. lettre 641, p. 435-436) avec une «combinaison canadienne» [publication de Monsieur Ouine] (lettre 642, p. 437-8) «fichtrement habilement rédigée» (lettre 643, p. 439). L'imposture ou le mercantilisme de Crespin fera son chemin jusque dans les Regards sur Bernanos de Henri Guillemin (Gallimard, 1976) où, significativement peut-être, la Librairie de France et les E.M.F. se confondent pour devenir «la Maison de France de New York» (p. 304).

7 . Ces données sont tirées de l'Almanach des Lettres - 1949, présenté par François Mauriac, Éditions de Flore et la Gazette des Lettres, p. 200-201. 
thêatre Marigny ${ }^{8}$. C'est dans ce contexte, avec cet arrière-plan politique et économique, que nous avons toujours lu et relu les textes de Robert Charbonneau sur la question du livre français publiés dans les livraisons de 1946 de la Nouvelle Relève, afin de mieux comprendre et apprécier le malaise du livre canadien-français et le problème de sa diffusion à l'extérieur du pays.

Remarquons-le bien. Le catalogue signalétique des Éditions France-Livre que nous rendons public aujourd'hui ${ }^{9} \mathrm{n}$ 'a rien à voir avec la notion ou le caractère de littérature canadienne; il n'est constitué en effet que d'imprimés canadiens. Le propriétaire-fondateur, M. Dorval, s'est clairement exprimé à ce sujet lors d'une entrevue où il racontait brièvement ses débuts de libraire-éditeur à un chroniqueur du Devoir, le 30 octobre 1948. Nous laissons au lecteur le soin d'en apprécier la logique:

M. Dorval à qui nous demandions ce qu'il pensait de la littérature canadienne, nous a admis en toute sincérité, qu' en autant que son commerce était concerné, les lecteurs de son quartier préféraient de beaucoup la littérature française à la littérature canadienne parce qu'ils lui trouvaient, ce qui manque encore à nos auteurs canadiens. De plus, ils conseillent à tous les jeunes gens de notre génération de lire les volumes techniques édités par Belisle de Québec, afin qu'ils découvrent l'aide que leur apporteront ces ouvrages dans la recherche d' une situation stable et rémunératrice.

En nous quittant, $M$. Dorval affirme sa confiance dans les auteurs canadiens, il croit à l'avenir de notre littérature à condition que le public veuille bien seconder les efforts de nos écrivains.

On en conviendra facilement, tous les titres de la maison France-Livre font partie de la petite littérature ou littérature de masse à l'exception sûrement de la réimpression de l'Arc de triomphe d'Erich-Maria Remarque.

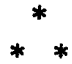

8 La Grande Pitié du livre français, Paris, Editions du Mail, p. 8-9: Le livre est notre nourriture spirituelle [...], il importe [...] de sauver le livre français, notre porte-parole le plus autorisé à l'étranger; et p. 31: C'est de France que doit part ir le livre français; L'introduction des livres français, édités en Suisse ou au Canada, sur le marché français, ne peut se faire que d'accord avec les auteurs et les éditeurs, et sous le contrôle du ministère des Affaires étrangères.

9 La présentation des notices de ce catalogue établi par Jacques Beaudry est inspirée des Règles de catalogage anglo-américaines, Montréal, ASTED, 1980. 


\section{CATALOGUE FRANCE-LIVRE (1944-1946)}

\section{Principaux éléments d'information pouvant apparaître dans une notice}

NOM, Prénom de l'auteur (Nationalité, ordre religieux, dates vitales, mention de pseudonyme). - Titre de l'ouvrage: complément du titre / mentions de responsabilités. - Lieu de publication: Editeur, date de publication, copyright (Lieu d'impression: Imprimeur, date de l'achevé d'imprimer). - Nombre de pages: autres caractéristiques matérielles; format (prix de vente). — («Titre de la collection»; numéro à l'intérieur de la collection). - Genre. - Mention de réimpression. - Diffusion. - Tirage. - Aperçu. - Contenu. - Sources. - Mention de descriptions. - Localisation.

\section{Abréviations, signes et symboles}

\section{Abréviations}

A. ach. d'impr. achevé d'imprimer

All.

av.-pr.

c

ca

$\mathrm{cm}$

coll.

coul.

couv.

C.R.

éd.

et al.

ex.

f.

F

ill.

$n \& b$

$n^{\circ}$

p.

p. de couv. page de couverture

photogr. photographie, $-s$

pseud.

pub.

R

RP

s.d.

suppl.

t.

Symboles
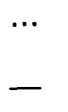

[notice]

\section{Allemand}

avant-propos

copyright

circa

centimètre, $-\mathrm{s}$

collection

couleur

couverture

compte rendu

édition

et autres

exemplaire

feuille, -s

Français

illustrateur, -s

illustration, $-\mathbf{s}$

noir et blanc

numéro

page, -s

pseudonyme

publicité

roman

roman populaire

sans date

supplément

tome, -s

pour signaler la suppression d'une partie d'un élément. est substitué aux points de suspension dans un titre.

la notice est placée entre crochets quand on n'a pas l'exemplaire en main; aucun crochet n'apparaît alors dans la notice.

[ ] interpolation d'une information quand on a l'exemplaire en main.

[ ?] interpolation incertaine

[ca 1960] date approximative

Sigles et abréviations (références et localisations)

DOLQ Dictionnaire des cuvres littéraire du Québec (Fides, 1978-1987)

Drolet Drolet, Antonio, Bibliographie du roman canadienfrançais, 1900-1950 (PUL, 1955)

Hare Hare, John E., Bibliographie du roman canadienfrançais, 1837-1962 (Fides, 1965)

NLFCCB B.N.Q., Notices en langues française du *Canadian Catalogue of Books 19211949» (M.A.C., 1975)

NUC The National Union Catalog

Vinet Vinet, Bernard, Pseudonymes québécois (Garneau, 1974).

MUTR Bibliothèque municipale de Trois-Rivières

UQTR Bibliothèque de l'Université du Québec à Trois-Rivières 
AMAT, J[ean] M[arie] d' ([F?]). - Malespine: roman / ill. [Léon Fauret?]. L- Montréal: France-Livre, 1945 (Imprimé au Canada). - 191 p., [f. d'errata]: ill. (n\&b), jaquette (coul.); $20 \mathrm{~cm}(1,25 \$)$. — Genre: RP. - Réimpression de l'éd. de: Paris: L'Illustration, c1937. - Argument: «Malespine est le nom de la famille très ancienne et très noble du héros de ce roman... "Traqués tantôt par les bandes des dauphins et tantôt par celles des rois de Sicile, écrasés en rase campagne, mais inexpugnables dans leurs montagnes, les sires de Malespine avaient fécondé de leur sang les quelques milliers d'hectares de bois et de landes dont ils revendiquaient la souveraineté totale." C'est sans doute à tout ce sang répandu que le dernier de la lignée, le marquis Rainier-Jean de Malespine, doit de poser... un personnage assez falot» - C. Hamel, «Malespine», le Canada, 13 mai 1946, p. 5. - Selon le NUC, le nom de l'A. serait J.C. d'Amat. - Source: ex. de la Collection Beaudry. L'ex. ne comprend pas l'errata. - Description dans: DOLQ III, p. 1138, à J.-M. Nemo; Drolet, no 50; Hare, p. 15; NLFCCB, p. 187; NUC. - Localisation: UQTR, PS8451A53M3 1945.

BAINVILLE, Jacques (F, 1879-1936). - Histoire de trois générations: avec un épilogue pour la quatrième. - Montréal: France-Livre, [ca 1944] (Imprimé au Canada). - 255 p.; $18 \mathrm{~cm}$ (1,25 \$). — Genre: Essai historique. - Réimpression de l'éd. de: Paris: Fayard, 1939, c1918. («Grandes Études historiques»). - Distribué par: France-Livre. - Argument: «On compte, en moyenne, trois générations par siècle. Presque tous ceux qui, en 1914, avaient atteint l'âge d'homme touchaient, par leurs grand-pères, à la période qui a suivi Waterloo. Ces cents années, qui ont mûri la plus grande guerre de tous les temps, qui sont si près de nous et qui nous ont faits ce que nous sommes, elles sont pourtant mal connues. Nous avons essayé d'en présenter un raccourci et une synthèse et de montrer la suite et le fil des événements» - av.-pr. - Sources: ex. MUTR; le Canada, 17 oct. 1944, p. 14, pub. et 5 févr. 1945, p. 7, “"L'égoïsme sacré" et la France», par H. Girard. — Localisation: MUTR, 944.06B162h.

[BERT, Jean (F?). - Fiancé du 1er avril. - Montréal: France-Livre, ca 1945. - $(0,10 \$)$. - («Mon livre favori»; 11). — Genre: RP. - Source: $4 c$ p. de couv. de le Document qui tue (1945), par John Canna.]

CANNA, John ([F?]). - le Document qui tue: roman policier. - Montréal: France-Livre, 1945 (Montmagny: Presses des Éditions Marquis, ach. d'impr. 12 déc. 1945). - 211, [1] p.; $20 \mathrm{~cm}(0,25 \$)$. - ([«Votre favori»; 20]). - Titre de la couv.: Don Sterling, détective. Le Document qui tue. - Genre: RP. - Source: ex. de la Collection Beaudry. A la fin de cet ex., trois premières p. d'un autre roman du même A., la Marque du crime.

[CANNA, John (F?). - la Marque du crime: roman policier. - Montréal: FranceLivre, ca 1945. - $20 \mathrm{~cm}$. - («Votre favori»; 21?). - Genre: RP. - Source: trois p. de cet ouvrage à la fin de l'ex. de le Document qui tue (1945) du même A., Collection Beaudry.]

[CATON, Henriette (F?). - À New-York - un soir. - Montréal: France-Livre, ca 1945. - (0,10\$). — («Votre favori»; 4). — Genre: RP. — Source: 4e p. de couv. de le Document qui tue (1945), par John Canna.]

[COUSINE ZO-ZO (F?, pseud. de J.M. d'Amat). - Guerlinguet le fâté. - Montréal: France-Livre, 1946. - 170, 3 p.; (1 \$). - Genre: Conte. - Résumé: "C'est l'histoire d'un écureuil qui entretient toute sorte de relations lafontainesques avec les petites bêtes de la création. Une histoire charmante pour les enfants qui sont à l'âge de la première communion et qui n'ayant pas lu M. Sartre ont le bonheur de n'avoir aucune de nos exigences existentialistes» - R.G. - Sources: le Canada, 3 déc. 1945, 
p. 8, «Des livres pour les jeunes», par R.G.; la Tribune, 30 nov. 1945, suppl. littéraire, p. 7, «Editions France-Livre» et photogr. de la couv. - Description dans: DOLQ III, p. 1138, à J.-M. Nemo; Drolet, no 49; Hare, p. 15; NLFCCB, p. 210; Vinet, p. 55.]

CROISSET, Francis de (F, Belge de naissance, 1877-1937, pseud. de Franz Wiener). - Nous avons fait un beau voyage: [aventures. Les Indes mystérieuses]. - Montréal: France-Livre, [1944]. — (1,25 \$). — Genre: Récit de voyage. — Réimpression de l'éd. de: Paris: Grasset, 1930. - Distributeur: France-Livre. - C.R.: «Le "beau voyage" de Croisset nous intéresse surtout parce qu'il nous met sous les yeux des spectacles auxquels nous ne sommes points accoutumés. Toutes ces glorieuses splendeurs des palais hindous, toute cette magnificence qui accompagne les chasses et les fêtes, tout le grouillement de ces métropoles où "des Canadiens passent, avec leurs belles gueules en ciment armé", tout cela ne fait de M. de Croisset qu'un peintre médiocre, mais toujours l'affirme comme l'homme du théâtre bourgeois d'un Paris qui n'est plus. Bien des gens ne retiendront de cet ouvrage que la condamnation explicite de l'attitude des Anglais à l'égard des Hindous. Mais Francis de Croisset voyageait aux Indes en 1929» - H. Girard, «Grand voyage aux Indes», le Canada, 4 déc. 1944, p. 9. - Source: ex. Coll. Beaudry et le Canada, 22 oct. 1945, p. iv, photogr. de la jaquette.

DANIEL, Élie ([F?]). - Serait-ce vraiment la fin des temps?: étude sur les prophéties.- Montréal: France-Livre, 1945 (Imprimé au Canada, ach. d'impr. 21 juil. 1945). - 448 p.; $20 \mathrm{~cm}$ (2,50 \$). — Genre: Étude. - Réimpression de l'éd. de: Paris: Téqui, 1931. - Résumé: La question de la fin du monde dans les prophéties scripturaires, la prophétie des papes et les prophéties modernes. Voir «La fin des temps», dans le Canada, 27 août 1945, p. 5. - Source: ex. UQTR. - Localișation: UQTR, CCSV BT875D36.

[DAUBRY, Jean (F?). - la Maison de la haine. - Montréal: France-Livre, ca 1945. - (0,10\$). - («Votre favori»; 15). - Genre: RP. - La coll. «Mon Livre favori» changerait, avec ce no, de titre pour devenir «Votre favori». - Source: $4^{e}$ p. de couv. de le Document qui tue (1945), par John Canna.]

[DAUBRY, Jean (F?). - la Douloureuse Destinée. - Montréal: France-Livre, ca 1945. - (0,10 \$). - («Votre favori»; 18). - Genre: RP. - Source: $4^{*}$ p. de couv. de le Document qui tue (1945), par John Canna.]

[DAZERGUES, Max-André (F?, pseud.). - lè Cocktail de minuit. - Montréal: France-Livre, ca 1945. - $(0,10 \$)$. - («Mon livre favori»; 1). — Genre: RP. — L'A. est membre de la Société des Gens de lettres. - «Mon livre favori», «collection d'amours, d'intrigues et de sensations» - source. - Source: $4^{\mathrm{e}} \mathrm{p}$. de couv. de le Document qui tue (1945), par John Canna.]

EN COLLABORATION. - la Vie et les æuvres de quelques grands saints: tome premier / Emile Baumann... [et al.]; av.-pr. Gabriel Mourey. - Montréal: France-Livre, [s.d.] (Montréal: Laplante \& Langevin). - 283, [2] p.; $21 \mathrm{~cm} \mathrm{(3} \mathrm{\$} \mathrm{pour} 2$ t.). - Genre: Hagiographie. - Réimpression de l'éd. de: Paris: Librairie de France, 1926. - Contenu: Saint Paul / Émile Baumann-Saint Augustin / Louis Bertrand-Saint Martin / René Dumesnil- Sainte Geneviève / André Pératé-Saint Rémi / Henri Moysset-Saint Benoît / René Schneider-Saint Bruno / Mario Meunier- Saint Bernard / René Guénon-Saint Dominique / Georges Bernanos-Saint François d'Assise / Gabriel Mourey - Sainte Claire / R. Havard de la Montagne- Saint Louis / Xavier de Magallon-Saint Bonaventure / Étienne Gilson-Saint Thomas d'Aquin / Jacques Maritain- Sainte Angèle de Foligno / Paul Bourdin. - Source: ex. de la Collection Beaudry. 
[EN COLLABORATION. - la Vie et les æuvres de quelques grands saints: tome deuxième / André Bellessort... et al. - Montréal: France-Livre (Montréal: Laplante \& Langevin). - 284, 1 p.; $21 \mathrm{~cm}$ ( $3 \$$ pour 2 t.). - Genre: Hagiographie. - Réimpression de l'éd. de: Paris: Librairie de France, 1926. - Contenu: Sainte Catherine de Sienne / Vallery-Radot-Saint Vincent Ferrier / René Johannet-Sainte Jeanne d'Arc / Stanislas Fumet-Saint Ignace / Gaëtan Bernoville-Saint François Xavier / André Bellessort-Sainte Thérèse d'Avila / Jean Soulairol-Saint Jean de la Croix / Maurice Brillant-Saint François de Sales / Henry Bordeaux - Sainte Jeanne Chantal / Isabelle Rivière-Saint Vincent de Paul / René Benjamin - Sainte Rose de Lima / Marcelle Mourey - Sainte Marguerite-Marie / Henri Ghéon-Saint Benoît-Joseph Laḅre / Charles Grolleau-Saint Jean-Baptiste Vianney / André Thérive-Sainte Thérèse de l'Enfant Jésus / André George. — Source: R. Houde et ex. de l'éd. Librairie de France.]

[HENRY, Eugène d' (F?). - la Marque de la honte. - Montréal: France-Livre, ca 1945. - (0,10\$). - («Mon livre favori»; 8). - Genre: RP. - Source: 4 e p. de couv. de le Document qui tue (1945), par John Canna.]

[JACQUES, Laurette (F?). - Mademoiselle Domino Rose. - Montréal: FranceLivre, ca 1945. - (0,10\$). - («Mon livre favori»; 9$)$. - Genre: RP. - Source: $4 \mathbf{c}$ p. de couv. de le Document qui tue (1945), par John Canna.]

[JAGOT, P.C. (F?, 1889-1962). — Comment on devient hypnotiseur. - Montréal: France-Livre, 1945. - (1 \$). - Genre: Livre pratique. — L'un des auteurs les plus appréciés de l'entre-deux guerres dans le domaine de la psychologie pratique et de la pratique du magnétisme, de l'hypnotisme, de la suggestion. - Réimpression de: $2^{\mathrm{e}}$ éd. Paris: Dangles, 194-?. - Source: le Canada, 22 janv. 1945, p. 9, pub. et 22 oct. 1945, p. iv, photogr. de la couv.]

MAGALI (F, pseud. de J.É.M.J. Corradot Philbert). — Un amour comme le nôtre: roman. - Montréal: France-Livre, 1944 (Imprimé au Canada). - 256 p.; $18 \mathrm{~cm}(1,25 \$)$. - Genre: RP. - Réimpression de l'éd. de: Paris: Tallandier, c1937. - Distribué par: France-Livre. - C.R.: «C'est donc beau l'amour! Surtout l'amour bien artificiel et sentimental, surtout l'amour qui n'existe que dans les romans idéalistes. Ah, ces monstres: Duhamel, Martin du Gard, Romains et tous les autres qui font dans la littérature! En ont-ils violé des imaginations pures! Heureusement, les petites gens de chez nous rejettent leurs écrits méchants et sceptiques. Il existe de bons auteurs qui, eux au moins ont trouvé l'art d'exprimer "les passions du cœur" comme elles doivent se comporter, de la façon qu'elles récompensent toujours la vertu et punissent toujours les traîtres. Sur ce terrain, Magali est imbattable. Elle enfonce Delly et M. Henry Bordeaux... Voici André Joranne, chirurgien naturellement célèbre et riche, d'un si beau caractère, d'une nature tellement exemplaire... [et Marie-Claude, une] jeune ingénue, fantasque et pourtant si intelligente, mutine, audacieuse et tellement timide... Le célèbre chirurgien, naturellement dégoûté de la vie parisienne, épouse dans un grand mouvement de passion cette fleur des bois. La femme fatale survient qui veut rompre ces tendres liens. Mais la providence de Magali est là qui ne permet point ces horribles choses... Pas de discussions, s'il vous plaît. Le roman affirme l'amour comme il devrait être. Et comment savez-vous que l'auteur n'a pas raison contre nous gens ricaneurs?»H. Girard, «Drame et chanson», le Canada, 25 sept. 1944, p. 5. — Source: ex. MUTR. - Localisation: MUTR, M188uac.

MAGALI (F, pseud. de J.É.M.J. Corradot Philbert). - Cour de flamme: roman. - Montréal: France-Livre, [1944] (Imprimé au Canada). - 255 p.; $18 \mathrm{~cm}(1,25 \$)$. - Genre: RP. - Réimpression de l'éd. de: Paris: Tallandier, c1933. - Distribué par: France-Livre. - Résumé: «C'est l'histoire d'une jeune fille riche qui aime un jeune 
homme pauvre» - «Magali», le Canada, 11 déc. 1944, p. 5. - Source: ex. relié MUTR. - Localisation: MUTR, M188cdf.

[MAGALI (F, pseud. de J.E.M.J. Corradot Philbert). - l'Armoire normande: roman. - Montréal: France-Livre,1944. - (1,25 \$). - Genre: RP. - Réimpression de l'éd. de: Paris: Tallandier, 1934. - Résumé: «Mona, depuis la mort de ses parents, demeure avec sa grand'mère et ses tantes... La petite est choyée on ne peut plus à une condition, toujours la même, cependant. En effet il faut que la petite obéisse aux vieilles, vieilles traditions qui sont loin d'une vie modernisée qu'elle voudrait vivre, l'ayant connue par les lectures. Les fréquentations quelles qu'elles soient sont interdites à la petite, devenue grande. Au moment solennel du conjugo, avec le Monsieur choisi par sa grand'mère... la jeune Mona reçoit la veille même de son mariage l'héritage de sa mère: ce n'est autre que la clef d'une Armoire, close à tout jamais, où étaient enfermé[es]... des lettres d'amour échangées entre sa mère et son père avant leur union. À leur lecture la jeune fille est bouleversée au point qu'elle quitte dans la nuit la demeure de sa grand'mère pour aller à Paris auprès d'une autre tante qui la reçoit à bras ouverts et qui connaissant tout le trouble de la jeune Mona la dirigera désormais» - le Canada, 19 févr. 1945. - Source: le Canada, 18 déc. 1944, p. 8, pub., 19 févr. 1945, p. 5, «L'armoire normande» et 22 oct., p. iv, photogr. de la couv.]

MAGali (F, pseud. de J.É.M.J. Corradot Philbert). — le Prisonnier de Bérangère: roman. - Montréal: France-Livre, [1945 ou 1946] (Montréal: Laplante et Langevin). - 256 p.; 19 cm. - Genre: RP. - Réimpression de l'éd. de: Paris: Tallandier, c1935. - Source: ex. MUTR. — Localisation: MUTR, M188pdb.

[MAGAli (F, pseud. de J.E.M.J. Corradot Philbert). - Pour devenir Lady. - Montréal: France-Livre, 1946. - (1,25 \$). - Genre: RP. - Source: le Canada, 18 mars 1946, p. 5, en annonce la parution prochaine.]

[MARQUISE DE CIRCÉ (F?). - l'Art de tirer les cartes. - Montréal: FranceLivre, 1945 ou 1946. - Genre: Livre pratique. - Source: 4e p. de couv. de le Prisonnier de Bérangère (1945 ou 1946), par Magali.]

[MARSELE, Claude (F?). - le Secret des vivants. - Montréal: France-Livre, ca 1945. - (0,10\$). - («Mon livre favori»; 3). - Genre: RP. - Source: 4c p. de couv. de le Document qui tue (1945), par John Canna.]

[MARSIEU, Paul (F?). — le Cœur pardonne. - Montréal: France-Livre, ca 1945. - (0,10\$). - («Votre favori»; 17). - Genre: RP. - Source: 4e p. de couv. de le Document qui tue (1945), par John Canna.]

[MONTEL, Claude (F?). - le Manoir aux volets clos. - Montréal: France-Livre, ca 1945. - (0,10\$). - («Votre favori»; 16). — Genre: RP. — Source: $4^{\circ}$ p. de couv. de le Document qui tue (1945), par John Canna.]

[MONTEL, Claude (F?). - Une heure d'amour. - Montréal: France-Livre, ca 1945. - (0,10\$). - («Votre favori»; 19). - Genre: RP. — Source: 4 c p. de couv. de le Document qui tue (1945), par John Canna.]

[MORIN, Ludovic (F?). - Domptée. - Montréal: France-Livre, ca 1945. - $(0,10 \$)$. - («Mon livre favori»; 6). - Genre: RP. — Source: 4e p. de couv. de le Document qui tue (1945), par John Canna.]

[MURIEL, Georges (F?). - À travers les barreaux. - Montréal: France-Livre, ca 1945. - (0,10\$). - («Mon livre favori»; 7). - Genre: RP. — Source: 4c p. de couv. de le Document qui tue (1945), par John Canna.] 
[NAMUR, Jean (F?). - le Destin vengeur. - Montréal: France-Livre, ca 1945. - $(0,10 \$)$. - («Mon livre favori»; 12). — Genre: RP. - Source: $4^{c}$ p. de couv. de le Document qui tue (1945), par John Canna.]

NEMO, Jean-Marie ([F?], pseud. de J.-M. d'Amat). - le Souffle du large: roman. - Montréal: France-Livre, 1945 (Montréal: Laplante \& Langevin, ach. d'impr. 20 déc. 1945). - 212 p.; $20 \mathrm{~cm}$ (1 \$). - Genre: RP. - Drolet, Hare et le DOLQ mentionnent comme éditeur «L'Imprimerie populaire» (!). - Avant l'ach. d'impr., cette mention: «New York 9 juin 1945». - Argument: «C'est là un simple petit roman de la mer, ou plutôt de deux marins qui se sont liés d'amitié et qui, tous deux, deviennent amoureux de la même femme» - «Le Souffle du large», le Canada, 4 nov. 1946, p. x. - Source: ex. UQTR. - Description dans: DOLQ III, p. 1138; Drolet, no 48; Hare, p. 15; NLFCCB, p. 217; Vinet, p. 182.

[REDANGES, Jacques (F?). - l'Amour d'un jour de fête. - Montréal: FranceLivre, ca 1945 . - $(0,10 \$)$. - («Mon livre favori»; 2$)$. - Genre: RP. - Source: $4^{e}$ p. de couv. de le Document qui tue (1945), par John Canna.]

[REMARQUE, Erich Maria Kramer (All., 1898-1970). - Arc de triomphe. - Montréal: France-Livre, 1945 ou 1946. - Genre: R?. - Source: le Canada, 22 oct. 1945 , p. iv, pub. qui en annonce la parution prochaine.]

SAGEHOMME, G. ([F?], s.j.,1862-1937). - Répertoire alphabétique de 10,000 auteurs avec 40,000 de leurs ouvrages (romans et pièces de théâtre) qualifiés quant à leur valeur morale. - Montréal: France-Livre, 1945 (Imprimé au Canada, ach. d'impr. 15 juil. 1945). - 643, [1] p.; $21 \mathrm{~cm}$ (2,50 \$). — Genre: Évaluation morale. Réimpression de l'éd. de: Paris: Casterman, 1939. - Tirage: comprend une éd. limitée avec suppl. spécial du R.P. Loubier, s.s.s., de Montréal. - Sources: ex. UQTR; le Canada, 17 oct. 1944, p. 14, pub. et 22, «France-Livre vivra». - Localisation: UQTR, ENT 15986.

[SALMON, Paul (F?). - Amour, Ó divin maître. - Montréal: France-Livre, ca 1945. - (0,10\$). - («Mon livre favori»; 5). — Genre: RP. - Source: 4 p. de couv. de le Document qui tue (1945), par John Canna.]

[STEPH (F?, pseud.). — Pâques de nos amours. - Montréal: France-Livre, ca 1945. - (0,10\$). - («Mon livre favori»; 10). - Genre: RP. - Source: 4 e p. de couv. de le Document qui tue (1945), par John Canna.]

[VERNE, Jules (F, 1828-1905). - Voyage autour de la terre. - Montréal: France-Livre, 1945 ou 1946. - (1 \$). - Genre: R d'anticipation scientifique. - Sources: $4^{\circ}$ p. de couv. de le Prisonnier de Bérangère (1945 ou 1946), par Magali; le Canada, 4 nov. 1946, p. x, pub.]

[VEUZIT, Max du (F,1886-1952, pseud. de Alphonsine Vavasseur-Acher Simonet). - John, le chauffeur russe. - Montréal: France-Livre, 1944. - (1,25 \$). - Genre: RP. - Réimpression de l'éd. de: Paris: Tallandier, 1931. - Aperçu: «Le roman d'une jeune fille moderne, orgueilleuse, luttant contre un amour qu'elle juge indigne de son immense fortune» - pub., le Canada, 17 oct. 1944, p. 14. - Source: le Canada, 1er mai 1944, p. 8, dans «Échos» et 22 oct. 1945, p. iv, photogr. de la couv.]

[VEUZIT, Max du (F,1886-1952, pseud. de Alphonsine Vavasseur-Acher Simonet). - l'Enfant des ruines. - Montréal: France-Livre, 1945. - (1,25 \$). - Genre: RP. - Réimpression de l'éd. de: Paris: Tallandier, 1938. - Aperçu: roman d'amour avec descriptions pittoresques et amusantes comme celle de la «fête religieuse et champêtre à la Madone le jour du Pardon - jour où les jeunes gens demandent à la Vierge Coquette de 
les guider dans le domaine matrimonial» - «France-Livre», le Canada, 9 avril 1945, p. 5. - Source: le Canada, 26 mars 1945, p. 5, pub. et 22 oct. 1945, p. iv, photogr. de la couv.]

WIRTA, Guy ([F?], 1897- , pseud.). — le Roi Jack. — [Montréal: France-Livre, 1944] (Imprimé au Canada). - 283 p.; $19 \mathrm{~cm}$ (1\$). — Genre: RP. - L'A. est membre de la Société des Gens de lettres. - Réimpression de l'éd. de: Paris, GauthierLanguereau, 1935, c1924. - Aperçu: «La lutte contre l'amour. La grandeur des sentiments qu'éprouve une jeune fille pour sa foi et son amour» - pub., le Canada, 17 oct. 1944, p. 14. - Sources: ex. de la Collection Beaudry; le Canada, 1er mai 1944, p. 8, dans «Echos». - Localisation: MUTR, W799r.

[YVELISE, Jean d' (F?, pseud.). - l'Appel d'un cœur blessé. - Montréal: France-Livre, ca 1945. - (0,10\$). — («Mon livre favori»; 13). — Genre: RP. — L'A. est membre de la Société des Gens de lettres. - Source: $4^{\mathrm{e}} \mathrm{p}$. de couv. de le Document qui tue (1945), par John Canna.]

[YVELISE, Jean d' (F?, pseud.). - la Robe blanche. - Montréal: France-Livre, ca 1945 . - (0,10\$). - («Mon livre favori»; 14). - Genre: RP. - L'A. est membre de la Société des Gens de lettres. — Source: 4e p. de couv. de le Document qui tue (1945), par John Canna.]

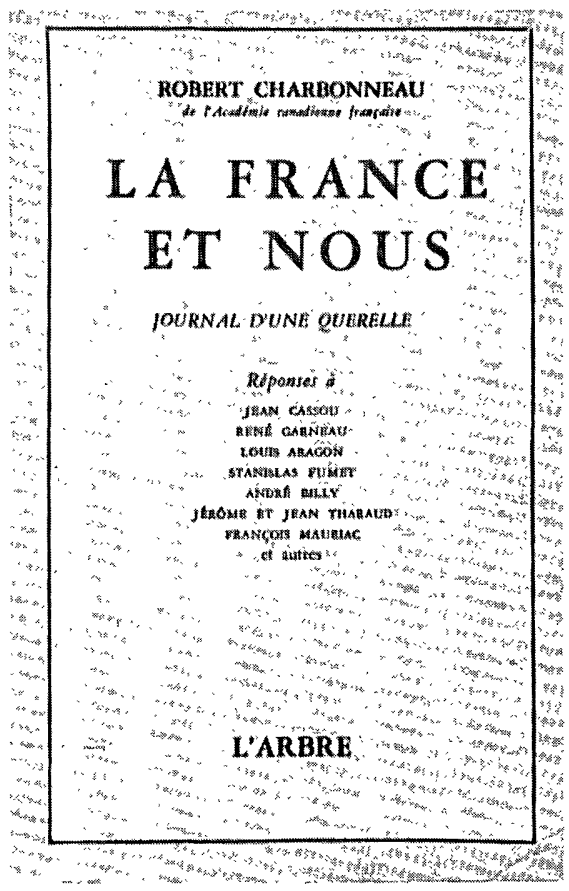

